\section{Auszeichnung für Asthma-Publikation}

\author{
Insgesamt 21 Arbeiten waren dieses Jahr zum Allergopharma \\ Award 2003 eingereicht worden. Am Ende setzte sich eine \\ Publikation von Dr. Peter Hellings vom Universitätskrankenhaus \\ Leuven in Belgien durch.
}

$\mathrm{D}$ er Allergopharma Award war im Jahr 2001 gemeinsam mit der European Academy of Allergology and Clinical Immunology (EAACI) ins Leben gerufen worden, um herausragende Forschungsergebnisse aus dem Bereich der allergischen Entzündung und der spezifischen Immuntherapie auszuzeichnen.

Der Preisträger 2003, Dr. Peter Hellings, wurde 1971 in Aalst geboren. Er studierte Medizin an der Katholischen Universität in Leuven und erhielt dort 1996 die Approbation. Er begann eine Facharztausbildung zum Hals-Nasen-Ohren-Arzt und führte Forschunmunology erschienenen ist. gen in der Arbeitsgruppe von Prof. Dr. Jan Ceuppens durch. Er promovierte 2001 mit einer Arbeit über „Immunomodulation in a mouse model of asthma“. Das Thema der allergeninduzierten Atemwegsentzündung in einem Mausmodell war auch Grundlage der jetzt ausgezeichneten Publikation „Blockade of CTLA- 4 enhances allergic sensitisation and eosinophilic airway inflammation in genetically predisposed mice", die im European Journal of Im-

Das T-Zell Oberflächenantigen CTLA-4 ist ein wichtiges co-stimulatorisches Molekül, das in die Herunterre-

\section{Heinz-Maurer-Preis}

Die Sebapharma GmbH \& Co. KG schreibt zum fünften Mal den Heinz-Maurer-Preis für hervorragende Forschungsarbeiten auf dem Gebiet der Dermatologie und angrenzenden Gebieten aus. Die Bereiche Grundlagenforschung und Klinische Forschung werden mit jeweils $5.000 €$ dotiert. Zugelassen sind wissenschaftliche Publikationen in deutscher oder englischer Sprache in anerkannten Fachzeitschriften, die im Zeitraum zwischen dem 1. Januar 2002 und dem 31. Dezember 2003 zum Thema „Hautoberfläche Modulation von Struktur und Funktion der Haut" veröffentlicht oder angenommen worden sind.

Von jedem Autor oder jeder Autorengruppe kann nur eine Arbeit eingereicht werden. Die eingereichte Arbeit darf nicht parallel bei anderen Ausschreibungen eingereicht worden sein. Die Preisträger räumen Sebapharma unentgeltlich das Recht ein, ihre Arbeit oder Auszüge daraus zu veröffentlichen oder anderweitig zu nutzen, sofern davon vorbestehende rechtliche Verpflichtungen nicht berührt werden. Ist die eingereichte Arbeit von mehreren Autoren verfasst, so wird der
Preis an den Erstautor verliehen. Die schriftliche Zustimmung der Mitautoren muss vorliegen.

Eine Kopie, ein Sonderdruck der eingereichten Publikation oder ein Manuskript mit beigefügter Publikationsannahmebestätigung durch die Redaktion der betreffenden Fachzeitschrift ist in zweifacher Ausfertigung bis zum 31. Dezember 2003 einzureichen. Detaillierte Ausschreibungsbedingungen sind online unter www.sebamed.de abzurufen.

Über die Verleihung des Heinz MaurerPreises für Dermatologische Forschung entscheidet eine Jury, bestehend aus den Mitgliedern des Wissenschaftlichen Beirates der Sebapharma und einem Firmenangehörigen.

\begin{tabular}{l}
\hline Sebapharma GmbH \& Co. KG \\
Frau Dr. Arens-Corell \\
Stichwort: Heinz-Maurer-Preis für Derma- \\
tologische Forschung \\
Postfach 1220 \\
56136 Boppard \\
Tel.: (o 6742 ) 9 oo-1 91 \\
Fax: (o 6742 ) 9 o0-1 86
\end{tabular}

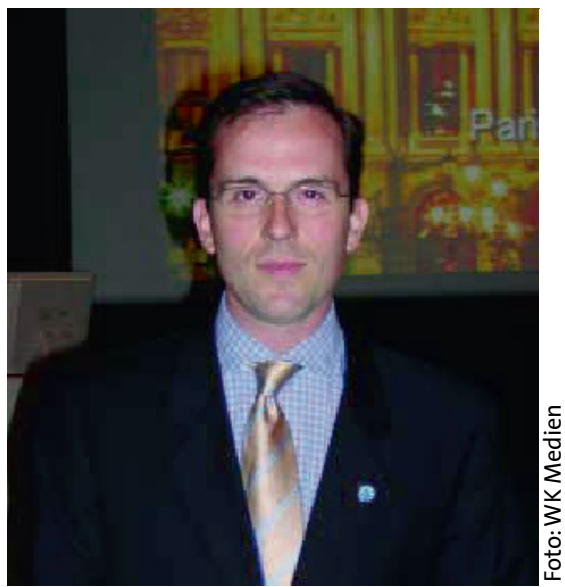

Dr. Peter Hellings, Preisträger des Allergopharma Award 2003

gulierung der T-Zell-Antwort involviert ist. Dr. Hellings und seine Kollegen zeigten, dass die gleichzeitige Gabe eines CTLA-4-neutralisierenden Antikörpers und eines sensibilisierenden Allergens die allergenspezifische IgEAntwort und Eosinophilie bei Mäusen mit einer genetischen Prädisposition zu einem allergischen Phänotyp erhöhte. Die Mäuse zeigten außerdem eine gesteigerte bronchiale Hyperreagibilität sowie erhöhte TH2-Zytokinspiegel in der broncho-alveolären Lavage, nach wiederholter Allergenprovokation auch reduzierte Spiegel an TGF- $\beta$. Die CTLA-4-Blockade blieb bei präsensibilisierten Mäusen sowie einem Mäusestamm ohne genetische allergische Prädisposition ohne Effekt.

Dr. Hellings und seine Kollegen folgerten daraus, daß die CTLA-4-Stimulation in einen wichtigen Regulationsmechanismus der allergischen Sensibilisierung in genetisch prädisponierten Mäusen involviert ist, möglicherweise vermittelt durch das Zytokin TGF- $\beta$. Es scheint so, dass die CTLA4-Blockade zum Zeitpunkt der Sensibilisierung zu einer Beeinträchtigung der Funktion und/oder Expansion der regulatorischen T-Zell-Population führen kann. Diese Beobachtungen bilden eine Basis, um vergleichbare Phänomene am Menschen zu untersuchen.

Prof. Dr. Paul van Cauwenberge, Präsident der EAACI, überreichte dem Preisträger das Zertifikat sowie einen Scheck über $10.000 € . \quad$ red 